(C) 2008 The Japan Society of Applied Physics

\title{
Size-Dependent Structural Characterization of Silicon Nanowires
}

\author{
Saeed AKhtar ${ }^{1}$, Koichi Usami ${ }^{1}$, Yoshishige TsuchiYa ${ }^{1,2}$, Hiroshi MizUta ${ }^{2,3}$, and Shunri Oda ${ }^{1,2 *}$ \\ ${ }^{1}$ Quantum Nanoelectronics Research Center, and Tokyo Institute of Technology, 2-12-1 O-okayama, Meguro-ku, Tokyo 152-8552, Japan \\ ${ }^{2}$ Solution Oriented Research for Science and Technology, JST, Kawaguchi, Saitama 332-0012, Japan \\ ${ }^{3}$ School of Electronics and Computer Science, Southampton University, Highfield, Southampton SO17 1BJ, U.K.
}

(Received November 22, 2007; accepted March 3, 2008; published online June 20, 2008)

Twin formation in silicon nanowires (SiNWs) grown via the vapor-liquid-solid (VLS) mechanism was investigated. We found that the formation of twins in SiNWs was predominantly affected by the SiNW diameter: they are formed only for SiNWs with a diameter larger than a certain critical value, while single crystal NWs can form when the diameter is smaller. Detailed observation revealed that, in SiNWs grown in the [111] direction, which are the most commonly observed NWs under current experimental conditions, periodic twins are formed while multiple twins are formed in the minor [110]-directed NWs. The defect formation mechanism is also discussed for the purpose of better control of crystal perfection in SiNWs. [DOI: 10.1143/JJAP.47.5053]

KEYWORDS: Si nanowires, VLS mechanism, $\mathrm{Si}_{2} \mathrm{H}_{6}$, low pressure chemical vapor deposition, Au droplets

\section{Introduction}

Semiconductor nanowire synthesis with superior crystal properties is a prerequisite for realizing nanowire based electronic and optoelectronic devices. ${ }^{1,2)}$ Silicon nanowires (SiNWs) are particularly promising for future nano-device applications. Over the past few years an extensive effort has been made toward controlled SiNW synthesis, mostly via the vapor-liquid-solid (VLS) mechanism. ${ }^{3-6)}$ Even though significant achievements have been made in the field of SiNW synthesis, ${ }^{7)}$ some fundamental questions remain, especially on the controlled growth of high-quality SiNWs. Controlled growth of high quality SiNWs is required for realizing device applications.

Until now, the VLS mechanism ${ }^{8,9)}$ of nanowire synthesis has proved to be the most tunable. The crystal quality of the wires grown by this technique can be very good and is usually considered "defect-free". In contrast, different types of defects have been observed in VLS-grown nanowires, ${ }^{10,11)}$ though single-crystal SiNWs have also been realized. Microscopic studies have revealed defects in SiNWs similar to other crystalline materials. ${ }^{12)}$ The investigations of these crystal defects are of vital interest as they will ultimately determine the performance of SiNW-based electronic or photonic devices. However, there is a lack of comprehensive understanding about defects and the defect-formation mechanism in SiNWs.

We have already reported the controlled-diameter $(12 \pm 5 \mathrm{~nm}) \mathrm{SiNW}$ synthesis by suppression of the aggregation of $\mathrm{Au}$ at $350^{\circ} \mathrm{C}$ by the VLS technique. ${ }^{13)}$ This technique has been realized by using the low-temperature decomposition of $\mathrm{Si}_{2} \mathrm{H}_{6}$.

In this paper we report details of the controlled-diameter $\mathrm{SiNW}$ synthesis at low temperatures from $\mathrm{Si}_{2} \mathrm{H}_{6}$ and the presence of defects in the wires grown by VLS-low pressure chemical vapor deposition (LPCVD). We demonstrate that the crystallinity of SiNWs is size-dependent. These wires preferentially grow along the [111] direction, whereas some SiNWs with the [110] growth direction have also been observed. We also present data showing periodic and multiple twin defects in SiNWs growing in the [111] and

*E-mail address: soda@pe.titech.ac.jp
[110] directions, respectively, with increasing diameters Moreover, studies show that the planar $\{111\}$ twins cut across the wires perpendicular to their growth direction and cause the side walls of the wires to be microfaceted. A twin formation mechanism in these wires is discussed.

\section{Experimental Procedure}

SiNW growth was carried out in an LPCVD chamber from $\mathrm{Si}_{2} \mathrm{H}_{6}$ using gold $(\mathrm{Au})$ droplets as a catalyst. The $\mathrm{Au}$ droplets were deposited using e-beam evaporation at room temperature. The average size distribution of the $\mathrm{Au}$ droplets was found to be $10 \pm 5 \mathrm{~nm}$ for an equivalent film thickness of $0.8 \mathrm{~nm}$ evaluated by a quartz crystal monitor. H-terminated $\mathrm{Si}(111)$ wafers with a resistivity $1.0-1.2$ $\Omega \cdot \mathrm{cm}$ were used as substrates. The SiNWs were grown by exposing the Au droplets covering $\mathrm{Si}$ substrates to a flow rate of $1 \mathrm{sccm}$ of $\mathrm{Si}_{2} \mathrm{H}_{6}$ and a flow rate of $49 \mathrm{sccm}$ of $\mathrm{H}_{2}$ at a pressure of 3 Torr. The Au droplets covering Si substrates were first annealed at a temperature of $375{ }^{\circ} \mathrm{C}$, measured at outer wall of the LPCVD chamber by thermocouple, and then exposed to the precursor gases for synthesizing SiNWs. The as-grown SiNWs were sonicated from the substrate into ethanol and were stretched across a lacey carbon grid to provide a vacuum background for transmission electron microscopy (TEM) images. Scanning electron microscopy (SEM) and TEM were used to analyze the samples.

\section{Results and Discussion}

Figure 1(a) is the SEM image of the Au droplets (10 \pm $5 \mathrm{~nm}$ ) used as a catalyst in this experiment. We observed that the crystalline SiNW synthesis can be realized by carefully controlling the $\mathrm{Au} / \mathrm{Si}$ ratio and the growth temperature. Figure 1(b) shows an SEM image of as-grown SiNWs grown at $375^{\circ} \mathrm{C}$ with diameters from $5-40 \mathrm{~nm}$. The wires are tens of micrometers long, where Fig. 1(c) shows that the average diameter of the wires is about $20 \mathrm{~nm}$. Growth temperature was found to be the key parameter affecting SiNW growth. Figures 1(d)-1(f) show the SEM images of SiNWs grown at $450{ }^{\circ} \mathrm{C}$, where the dense wires were partially grown only in varying size spheres [Fig. 1(d)] on the substrate with large and random diameter distribution $(400 \pm 50 \mathrm{~nm})$ as shown in the high-resolution SEM image in Fig. 1(e), whereas vertical Si micro pillars [Fig. 1(f)] were observed on rest of the 


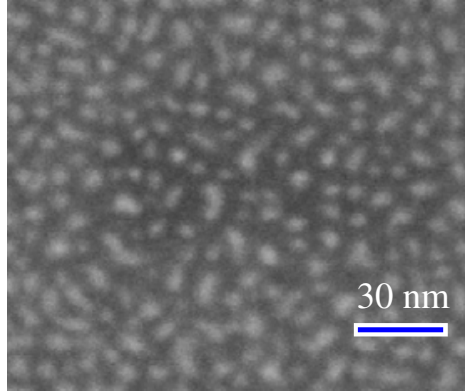

(a)

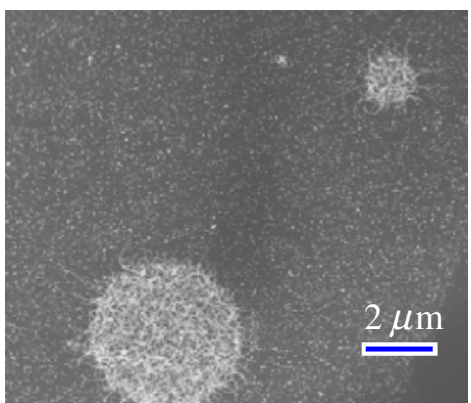

(d)

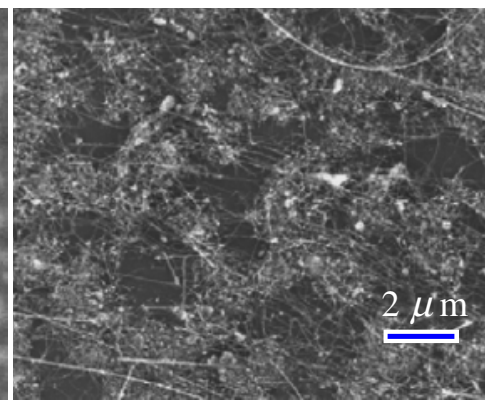

(b)

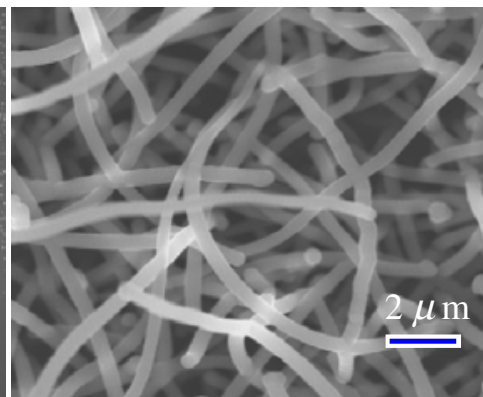

(e)

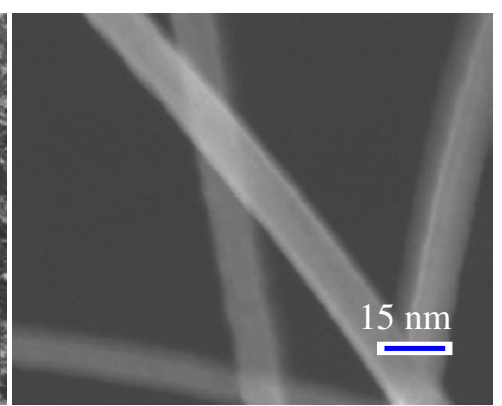

(c)

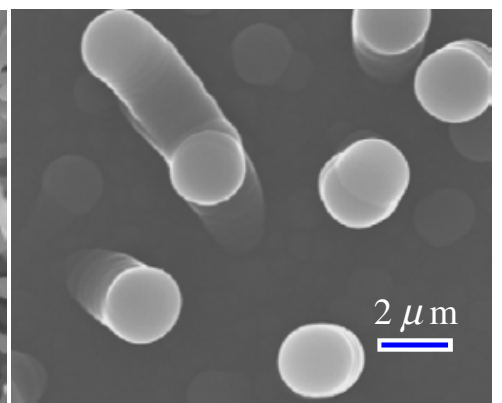

(f)

Fig. 1. (Color online) SEM images of (a) Au droplets prepared by e-beam evaporation at room temperature, (b) SiNWs grown at $375^{\circ} \mathrm{C}$, (c) a high-resolution image of a few SiNWs shown in (b), (d) SiNWs grown at $450^{\circ} \mathrm{C}$, and (e)-(f) high-resolution images of the partially-grown NWs in spheres and a large quantity of Si micropillars, respectively, shown in (d).

substrate. This temperature dependent mobility of the eutectic droplets also affects the structural properties of the wires. SiNWs were grown mostly via the VLS method. However, some wires could have grown via the base growth mechanism or by direct decomposition of $\mathrm{Si}_{2} \mathrm{H}_{6}$.

The as-grown SiNWs predominantly exhibit the [111] growth direction. A few wires with the [110] growth direction were also observed. It has been reported that the smallest-diameter SiNWs prefer to grow along the [110] growth direction. ${ }^{14)}$ We found that the growth direction is independent of the diameter of the wires.

The preferred direction of the single-crystal growth distinguishes between various VLS synthesis schemes for semiconductor nanowires. The semiconductor nanowires grown by laser ablation or CVD exhibit predominantly the [111] growth direction, ${ }^{3)}$ whereas wires prepared by the oxide-assisted technique prefer the [112] growth direction. ${ }^{15)}$ However, SiNWs produced by this technique grow along the [111] as well as the [110] direction.

Figures 2(a) and 2(b) are TEM images of the SiNWs with diameters about 6 and $5 \mathrm{~nm}$, respectively, having highquality single-crystal cores. These wires exhibit the [111] and [110] growth directions, respectively. These small-sized wires have very thin surface oxide less than $1 \mathrm{~nm}$ thick. SiNWs with the [110] growth direction were observed to be less than $2 \%$ of the total yield. TEM investigations have revealed that the SiNWs with diameters below $16 \mathrm{~nm}$ are mostly defect-free and single crystal.

On the other hand, a high density of twin defects was observed in wires with diameters larger than $16 \mathrm{~nm}$. A single grain boundary, indicated by an arrow in Fig. 3(a), in the form of a faint line was observed in the center of the SiNWs with diameters mostly above $17 \mathrm{~nm}$. Large numbers of twin defects in SiNWs with diameters above

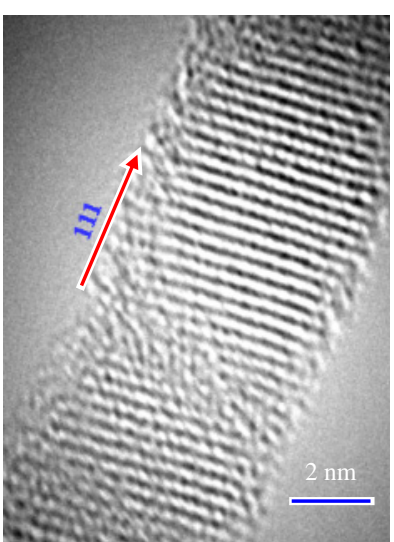

(a)

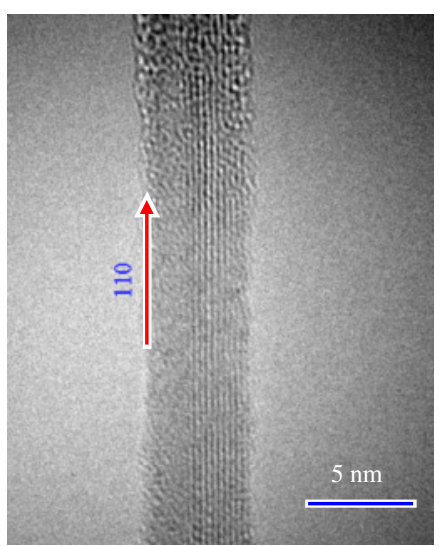

(b)
Fig. 2. (Color online) A high-resolution TEM image of SiNWs with diameters of about (a) 6 and (b) $5 \mathrm{~nm}$.

$20 \mathrm{~nm}$ were observed. These are periodic and multiple twins occurring in the wires depending upon the growth direction. Figure 3(b) is the high-resolution TEM image of a SiNWs exhibiting the [110] growth direction, determined from analysis of the lattice fringes, that has multiple $\{111\}$ twins. The diameter of the wire is about $25 \mathrm{~nm}$. A similar kind of twin defect has already been observed in SiNWs grown in the [112] and the [110] directions. ${ }^{16,17)}$ However, we observed that multiple twin defects only occur in SiNWs grown in the [110] direction, whereas periodic twins were observed only in the wires with the [111] growth direction. Such defects are termed lameller twins. ${ }^{18)}$ These twins exist in the entire length of the wires [Fig. 3(c)] and terminate at the interface with a eutectic alloy [Fig. 3(d)]. This suggests that these defects originate during nucleation and propagate into the wires. Such wires 


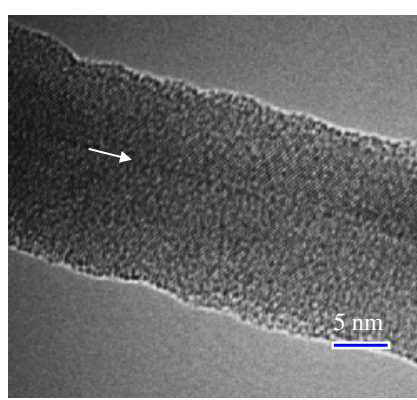

(a)

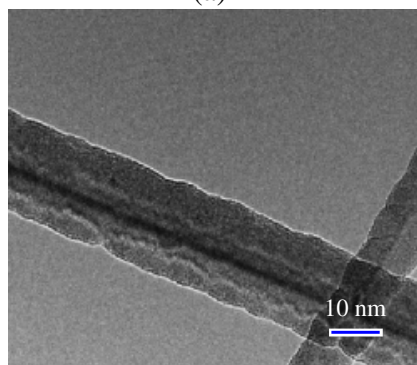

(c)

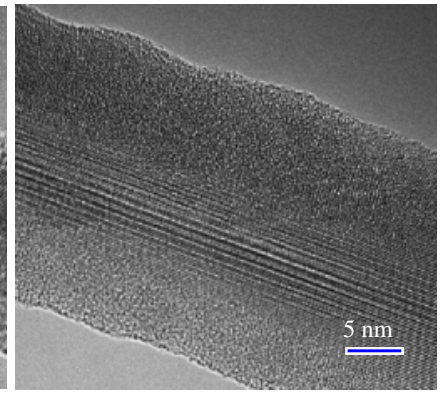

(b)

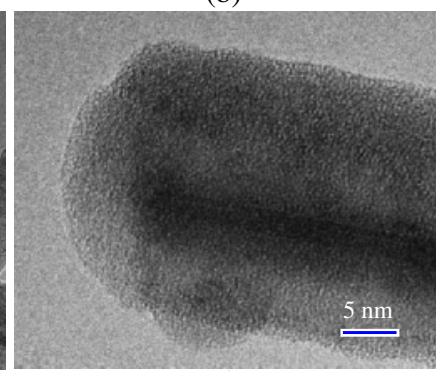

(d)
Fig. 3. (Color online) High-resolution TEM photographs of SiNWs showing structural distinctions between the SiNWs containing (a) a single twin boundary, and (b)-(d) multiple twin defects.

have a surface oxide about $8 \mathrm{~nm}$ thick with a non-uniform crystalline core.

We also propose that the initial nucleation influences the structural properties of the wires. ${ }^{18)}$ Since SiNWs are crystalline, the crystallization occurs epitaxially at the interface between the metal seed and the wire. The atomic layers grow at the $\mathrm{Au}-\mathrm{SiNW}$ interface and repeat the same crystal order in the entire length of the wire. The large-sized $\mathrm{Au}$ droplets nucleate heterogeneously resulting in the formation of multiple twins in the wires.

However, periodic twins were observed in SiNWs with the [111] growth direction. These twins bisect the wires perpendicular to their growth direction causing the side walls of the wires to be microfaceted [Fig. 4(a)]. Figure 4(b) shows a high-resolution TEM image of a SiNW containing a twin defect, which is indicated by an arrowhead. The diameter of the wires is about $22 \mathrm{~nm}$. The twinning period in these wires could not be measured as reported elsewhere. ${ }^{11)}$ The twins extend across the entire diameter of the wire as planer defects. The twin-plane formation in SiNWs grown in the [111] direction is quite expected because of the low twin energy for $\mathrm{Si}$ of about $22 \mathrm{meV} /$ bond or $28 \mathrm{~mJ} / \mathrm{m}^{2}{ }^{19}$ ) Therefore, it is common that $\{111\}$ twins form in SiNWs exhibiting the [111] growth direction.

Understanding the growth mechanism is necessary for realizing high-quality $\mathrm{SiNWs}$ from $\mathrm{Si}_{2} \mathrm{H}_{6}$. The epitaxial nucleation of the wires by this method can be divided into the following three steps (Fig. 5):

(i) the incorporation of $\mathrm{Si}$ into $\mathrm{Au}$ droplets from the underlying substrate resulting in molten $\mathrm{Au}-\mathrm{Si}$ eutectic alloy formation;

(ii) the cracking down and diffusion of source precursors on $\mathrm{Au}$ droplets; and

(iii) the diffusion of source precursors on Si substrate.

The molten eutectics are formed in step (i) during annealing of the $\mathrm{Au}$ that covers $\mathrm{Si}$ substrates [Fig. 5(a)].

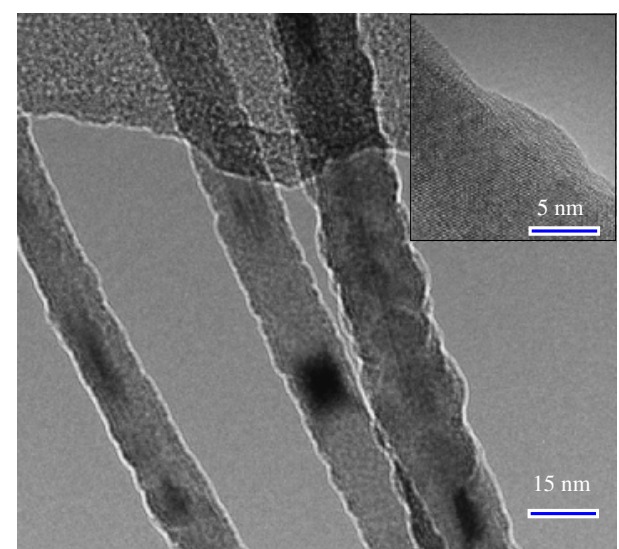

(a)

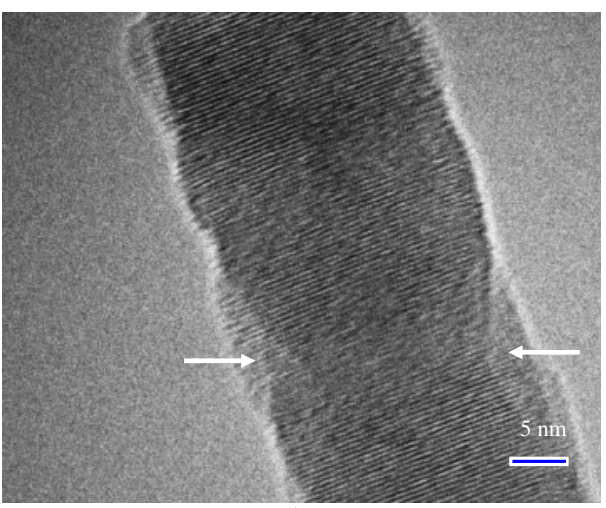

(b)

Fig. 4. (Color online) TEM images showing (a) zig-zag morphology of the SiNWs with a high-resolution view given in the inset, and (b) periodic twins in the SiNWs indicated with arrow heads.

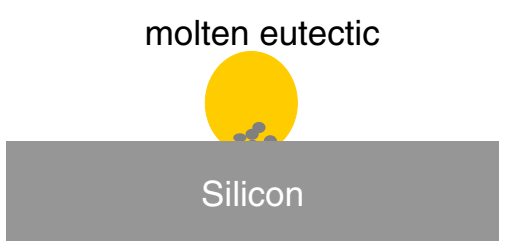

(a)

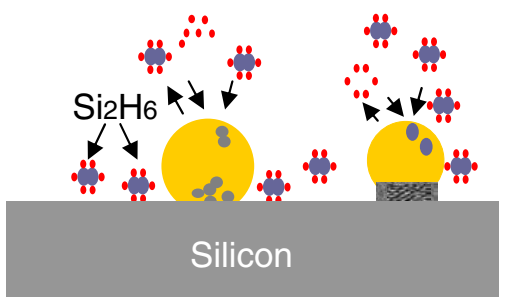

(b)

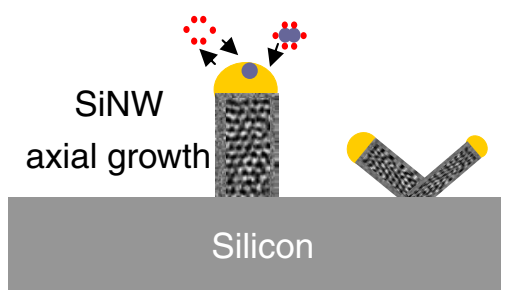

(c)

Fig. 5. (Color online) A schematic cross-section of (a) molten alloy formed upon annealing (yellow represents the Au droplets), (b) impingement of $\mathrm{Si}$ precursors (vapors of disilane, $\mathrm{Si}_{2} \mathrm{H}_{6}$, represented with red and gray), and (c) axial growth of SiNWs. 
When these eutectics are exposed to the precursor gases, steps (ii) and (iii) occur [Fig. 5(b)] contributing simultaneously to SiNW growth [Fig. 5(c)]. These nucleation stages play a key role in determining the growth mechanism, morphology, size distribution, and defect-formation in SiNWs. In step (ii) precursors diffuse into the eutectic alloys and take part in the axial growth of SiNWs after supersaturation. The impingement of the source precursor on the substrate is assumed to be uniform. We suggest that these precursors also diffuse on the substrate and reach a favorable eutectic site for contributing to SiNW nucleation. If no such site is found within a certain time, then they will desorb. This step also influences the movement of the eutectics on the substrates as a part of the catalyst aggregation. It could also be a probable cause of some base-growth mechanism and defect-formation due to the movement of the eutectics during SiNW nucleation.

The SiNW defects are diameter dependent and can be controlled by selecting a certain $\mathrm{Au} / \mathrm{Si}$ ratio, which depends on the amount of the catalyst and the annealing conditions. The shape of the $\mathrm{Au}-\mathrm{Si}$ eutectic is also temperature dependent and remains circular at low annealing temperatures. ${ }^{20)}$ We presume that these twin defects are formed due to fluctuations at the eutectic-SiNW interface caused by probable variations in the source gas concentration, temperature, movement of eutectic droplets on the substrates, or some other growth parameter. These fluctuations cause the wires to twin and kink and also influence their growth direction, for example, the transformation of the growth direction of the wires from the [111] to a bicrystalline ${ }^{13)}$ or the $\langle 112\rangle$ growth directions. ${ }^{18)}$ The bending of the wires under intrinsic stress could also cause twin formation. We believe that the eutectic droplets below $16 \mathrm{~nm}$ in size can accommodate fluctuations at the eutectic-SiNW interface that do not support twinning for a particular crystallographic direction. We propose that because of this, small diameter SiNWs do not exhibit twinning, whereas the large size eutectics form twins in the wires. The small size wires exhibit the lowest-energy (111) and (110) growth planes which also do not favor twin formation. This suggests that defect-free SiNWs can be realized by selecting a specific catalyst size, annealing conditions, and by maintaining the stability of the catalyst on the substrates.

\section{Conclusions}

In conclusion, we have investigated the twin formation mechanism in SiNWs grown at low temperatures via VLS-
LPCVD. Crystal-quality of the SiNWs strongly depends on the diameter of the wires which can be controlled using a certain catalyst size and growth conditions. The smalldiameter SiNWs are found to be free of defects whereas a high density of twins occurs in the large-diameter SiNWs with periodic twins in the wires exhibiting the [111] and multiple twins in the wires exhibiting the [110] growth directions. The defect formation mechanism in SiNWs based on the initial nucleation of the wires has been discussed to provide a better understanding for realizing defect-free SiNWs in a low temperature and low pressure growth process. Single-crystal SiNWs grown predominantly in the [111] direction with smaller diameters are attractive for electronic and optoelectronic applications.

1) Y. Huang, X. Duan, and C. M. Lieber: Small 1 (2005) 142.

2) J. Goldberger, A. I. Hochbaum, R. Fan, and P. Yang: Nano Lett. 6 (2006) 973.

3) Y. Cui, L. J. Lauhon, M. S. Gudiksen, J. Wang, and C. M. Lieber: Appl. Phys. Lett. 78 (2001) 2214.

4) D. D. D. Ma, C. S. Lee, F. C. K. Au, S. Y. Tong, and S. T. Lee: Science 299 (2003) 1874.

5) J. B. Hannon, S. Kodambaka, F. M. Ross, and R. M. Tromp: Nature 440 (2006) 69.

6) S. Hofmann, C. Ducati, R. J. Neill, S. Piscanes, A. C. Ferrari, J. Geng, R. E. Duin-Borkowaski, and J. Robertson: J. Appl. Phys. 94 (2003) 6005.

7) A. I. Hochbaum, R. H. R. Fan, and P. Yang: Nano Lett. 5 (2005) 457.

8) R. S. Wagner and W. C. Ellis: Appl. Phys. Lett. 4 (1964) 89.

9) E. I. Givargizov: J. Cryst. Growth 31 (1975) 20.

10) F. M. Davidson III, A. D. Schricker, R. J. Wiacek, and B. A. Korgel: Adv. Mater. 16 (2004) 646.

11) Q. Li, X. Gong, C. Wang, J. Wang, K. Ip, and S. Hark: Adv. Mater. 16 (2004) 1436.

12) Q. Tang, X. Liu, T. I. Kamins, G. S. Solomon, and J. S. Harris: Appl. Phys. Lett. 81 (2002) 2451.

13) S. Akhtar, K. Usami, Y. Tsuchiya, H. Mizuta, and S. Oda: Appl. Phys. Express 1 (2008) 014003.

14) Y. Wu, Y. Cui, L. Huynh, C. J. Barrelet, D. C. Bell, and C. M. Lieber: Nano Lett. 4 (2004) 433.

15) Y. F. Zhang, Y. H. Tang, N. Wang, C. S. Lee, I. Bello, and S. T. Lee: Phys. Rev. B 359 (2000) 4518.

16) N. Wang, Y. H. Tang, Y. F. Zhang, C. S. Lee, and S. T. Lee: Phys. Rev. B 58 (1998) R16024.

17) T. Hanrath and B. A. Korgel: Adv. Mater. 15 (2003) 437.

18) F. M. Davidson III, D. C. Lee, D. D. Fanfair, and B. A. Korgel: J. Phys. Chem. C 111 (2007) 2929.

19) B. Ressel, K. C. Prince, S. Heun, and Y. Homma: J. Appl. Phys. 93 (2003) 3886.

20) S. Takeuchi and K. Suzuki: Phys. Status Solidi A 171 (1999) 99. 